\title{
A influência do Transtorno do Déficit de Atenção e Hiperatividade (TDAH) no aprendizado de crianças: Uma Revisão Integrativa da Literatura
}

The influence of Attention Deficit Hyperactivity Disorder (ADHD) on children's learning: An

\author{
Integrative Literature Review
}

La influencia del trastorno por déficit de atención con hiperactividad (TDAH) en el aprendizaje de

los niños: Una revisión de la literatura integradora

Recebido: 20/05/2021 | Revisado: 29/05/2021 | Aceito: 03/06/2021 | Publicado: 18/06/2021

\author{
Luciana Vargas Pedroso \\ ORCID: https://orcid.org/0000-0003-2965-3083 \\ Universidade Federal do Pampa, Brasil \\ E-mail: lucianapedroso@unipampa.edu.br \\ Susane Graup \\ ORCID: https://orcid.org/0000-0002-3389-8975 \\ Universidade Federal do Pampa, Brasil \\ E-mail: susanegraup@unpampa.edu.br \\ Rodrigo de Souza Balk \\ ORCID: https://orcid.org/0000-0001-5254-6732 \\ Universidade Federal do Pampa, Brasil \\ E-mail: rodrigobalk@unipampa.edu.br \\ Carine Jardim de Castro \\ ORCID: https://orcid.org/0000-0001-9935-6178 \\ Universidade Federal do Pampa, Brasil \\ E-mail: carine.jcatro@gmail.com \\ Marcia Helena Rodrigues de Freitas Arend \\ ORCID: https://orcid.org/0000-0002-5350-9498 \\ Universidade Federal do Pampa, Brasil \\ E-mail: marciaarend@hotmail.com
}

\begin{abstract}
Resumo
Objetivo: O presente estudo tem como objetivo analisar como o TDAH interfere no desenvolvimento e aprendizagem das crianças na sala de aula, a partir de uma revisão de literatura, achados referentes à aprendizagem de crianças que possuem o transtorno e o quanto conseguem de fato assimilar os conteúdos, em processos de ensino-aprendizagem. Metodologia: Trata-se de um estudo de revisão integrativa da literatura, no qual foram realizadas buscas na base de dados LILACS e biblioteca virtual SCIELO. A combinação de descritores, com base nos Descritores em Ciências da Saúde - DeCS, utilizados para a busca foram "Transtorno do déficit de atenção e hiperatividade", "criança", "escolares", "docentes" e "professores", utilizando na busca os operadores booleanos AND e OR. Utilizou-se um recorte temporal de 1996 a 2020 nas buscas. Foram encontrados 646 trabalhos, porém somente 44 contemplaram os critérios de inclusão para análise na íntegra, resultando em 7 para compor a revisão. Foram direcionadas para a discussão duas categorias: a aprendizagem de crianças com TDAH e a percepção dos professores em relação ao transtorno. Conclusão: De modo geral, os estudos evidenciam que crianças com o transtorno apresentam dificuldades de aprendizagem e baixo rendimento escolar. Essa desordem, inegavelmente, interfere significativamente no processo ensino-aprendizagem de crianças na fase escolar tornando uma barreira para um bom desempenho acadêmico.
\end{abstract}

Palavras-chave: Transtorno do déficit de atenção e hiperatividade; Criança; Escolares; Estudantes; Professores.

\begin{abstract}
Objective: The present study aims to analyze how ADHD interferes in the development and learning of children in the classroom, based on a literature review, findings related to the learning of children who have the disorder and how much they are in fact able to assimilate. contents, in teaching-learning processes. Methodology: This is an integrative literature review study, in which searches were performed in the LILACS database and the virtual library SCIELO. The combination of descriptors, based on the Health Sciences Descriptors - DeCS, used for the search were "Attention deficit hyperactivity disorder", "child", "schoolchildren", "teachers" and "teachers", used in the search the Boolean operators AND and OR. A time frame from 1996 to 2020 was used in the searches. 646 papers were found, but only 44 met the inclusion criteria for full analysis, resulting in 7 to compose the review. Two categories were directed to the discussion: the learning of children with ADHD and the teachers' perception of the disorder. Conclusion: In general, studies show that children with the disorder have learning difficulties and low school performance. This
\end{abstract}


disorder undeniably interferes significantly in the teaching-learning process of children in the school phase, making it a barrier to good academic performance.

Keywords: Attention deficit hyperactivity disorder; Child; Schoolchildren; Students; Teachers.

\begin{abstract}
Resumen
Objetivo: El presente estudio tiene como objetivo analizar cómo el TDAH interfiere en el desarrollo y aprendizaje de los niños en el aula, a partir de una revisión de la literatura, hallazgos relacionados con el aprendizaje de los niños que padecen el trastorno y cuánto son de hecho capaces de asimilar contenido, en los procesos de enseñanza-aprendizaje. Metodología: Se trata de un estudio de revisión integradora de la literatura, en el que se realizaron búsquedas en la base de datos LILACS y la biblioteca virtual SCIELO. La combinación de descriptores, basados en los Descriptores de Ciencias de la Salud - DeCS, utilizados para la búsqueda fueron "Trastorno por déficit de atención con hiperactividad", "niño", "escolares", "profesores" y "profesores", utilizados en la búsqueda los operadores booleanos Y y OR. En las búsquedas se utilizó un período de tiempo de 1996 a 2020. Se encontraron 646 artículos, pero solo 44 cumplieron los criterios de inclusión para el análisis completo, lo que resultó en 7 para redactar la revisión. Dos categorías se dirigieron a la discusión: el aprendizaje de los niños con TDAH y la percepción del trastorno por parte de los profesores. Conclusión: En general, los estudios muestran que los niños con el trastorno tienen dificultades de aprendizaje y bajo rendimiento escolar. Este trastorno indudablemente interfiere de manera significativa en el proceso de enseñanza-aprendizaje de los niños en la etapa escolar, lo que lo convierte en una barrera para el buen rendimiento académico.
\end{abstract}

Palabras clave: Transtorno por déficit de atención com hiperactividad; Ninõ; Escolares; Estudiantes; Maestros.

\title{
1. Introdução
}

O Transtorno do Déficit de Atenção e Hiperatividade (TDAH) é definido como uma alteração neurobiológica de causas genéticas que aparece na infância e pode permanecer na vida adulta em $60 \%$ dos casos, com prejuízos no desempenho escolar (Abda, 2019; Miranda et al., 2016), na baixa performance profissional, na perda da renda familiar e no baixo repertório social (Larroca \& Domingo, 2012).

Dentre as principais características, o Manual Diagnóstico e Estatístico de Transtornos Mentais, destaca a impulsividade, a desatenção e a hiperatividade (DSM-V, 2014). De maneira geral, a criança com TDAH apresenta comportamentos comuns da infância, porém mais intensos e com facilidade de distração com as coisas ao seu redor, o que dificulta a concentração, a organização e a percepção dos detalhes, podendo ainda, apresentar relutância em realizar tarefas que lhes prenda a atenção por muito tempo e exijam esforço mental (Barkley, 2002; Mattos, 2006). Ainda de acordo com os autores, as crianças com TDAH não suportam coisas monótonas e repetitivas e não conseguem controlar seus impulsos.

As prevalências variam de acordo com o local do estudo. Nesse sentido, uma pesquisa realizada com crianças em idade escolar dos Estados Unidos, identificou índices entre 5\% a 7\% (Polanczyk et al., 2014). No Brasil, a pesquisa de Pastura et al. (2017), mostrou que as prevalências desse transtorno em escolares variaram de 5,8 a 17,1\%, evidenciando uma frequência alta de alunos com TDAH incluídos nas classes regulares de ensino. Nesse contexto, a escola precisa estar preparada para o atendimento desses alunos, mesmo que na ótica da educação inclusiva, os alunos com essas características não estejam incluídos na Educação Especial. Assim, o ensino de crianças com esse problema pode ser considerado algo desafiador para os profissionais da educação que podem apresentar falta de entendimento e manejo adequado com crianças que possuem esse transtorno, devido a lacunas no processo de formação dessa categoria acerca da educação inclusiva e no desenvolvimento de relações entre a teoria e a prática que possam atender as especificidades educacionais desses aprendizes (Moura, Silva \& Silva, 2019; Silva, 2015).

Com a falta de preparo das escolas e dos professores para identificarem as necessidades especiais dos alunos com TDAH, no que diz respeito à aprendizagem, percebe-se fragilidades no desenvolvimento do aluno. A esse respeito, Gonçalves e Volk (2016), afirmam que para esse estudante desenvolver sua autonomia e potencialidades, os processos educativos devem ir ao encontro de suas necessidades.

Considerando a escola uma das principais instituições de inserção social das crianças, estas têm o papel fundamental 
de atender toda e qualquer diferença individual dos sujeitos. Portanto, o ambiente acolhedor propicia as diversidades dos alunos, assim como a demanda contemporânea cria novas práticas e estratégias de ensino adequadas (Zucchetti, 2011; Barbarini, 2020). Dessa forma, quando os processos de ensino são adequados, as crianças se tornam rapidamente protagonistas de sua maneira de desenvolvimento social. O uso apropriado de intervenções potencialmente relevantes e voltadas a essas crianças tornam-se necessárias e visam diminuir ocorrências de atitudes que possam prejudicá-las, auxiliando de maneira que minimize o sofrimento e potencialize o seu bem estar (Barbarini, 2020).

Vale destacar que as estratégias adequadas de ensino, como por exemplo, acomodar os alunos próximo ao professor (mesa bem da frente), longe de janelas e portas evitando distrações, realizar atividades dinâmicas e de entretenimento de curta duração, implantar a organização de materiais e tarefas no final da aula visando a obtenção de uma rotina para a revisão e aquisição de conteúdos, bem como elogiar as crianças durante a participação em sala de aula podem favorecer o aprendizado dos estudantes que possuem o transtorno (Mattos, 2006). As crianças são capazes de aprender e atingir resultados satisfatórios, porém com bastante dificuldade e cada uma no seu tempo, muitas vezes precisando de um tempo maior para compreender e internalizar os conteúdos devido alguns fatores associados que podem ser responsáveis pelo péssimo rendimento escolar, assim como prejuízos na vida social, familiar e profissional (Maia \& Confortin, 2015; Silva, et al., 2020).

A aprendizagem é identificada em três tipos gerais como a cognitiva, afetiva e psicomotora, porém no ensino aborda a humanística, comportamentalista e cognitivista. Ao aprender, a criança atribui significados na sua realidade atual estabelecendo relações de significação e atuação no processo de aprendizagem (Moreira, 1999). Diante dessas informações, o objetivo do presente estudo é analisar como o TDAH interfere no desenvolvimento e aprendizagem das crianças na sala de aula.

\section{Metodologia}

Trata-se de uma revisão integrativa da literatura e tem a finalidade de condensar os estudos já realizados por outros autores com o propósito de aprofundar o conhecimento científico sobre o tema investigado e contribuir para o planejamento de futuros estudos. Para a elaboração desse estudo foram utilizadas as seguintes etapas preconizadas por Mendes, Silveira \& Galvão (2008): I) Estabelecimento da hipótese ou questão da pesquisa; II) Busca na literatura; III) Categorização dos estudos; IV) Avaliação dos estudos incluídos na revisão; V) Interpretação dos resultados; VI) Síntese do conhecimento.

Seguindo as etapas metodológicas, na primeira fase do estudo, em relação à questão norteadora foi desenvolvida pelo plano PICo que se refere a um acrossílabo cujo significado é participante, fenômeno de interesse e contexto e que, nesse caso, "P" crianças, "I" interferência do TDAH no desenvolvimento e aprendizagem das crianças que possuem o transtorno e "Co" sala de aula. A questão norteadora foi: "O TDAH interfere no desenvolvimento e aprendizagem das crianças na sala de aula?".

$\mathrm{Na}$ segunda fase, iniciou-se a busca e seleção em bases de dados online, durante o mês de abril de 2021. Como critérios de inclusão foram incluídos na revisão: artigos originais disponíveis na íntegra, em meio online, artigos publicados em idiomas português, inglês ou espanhol. O período utilizado para o recorte temporal foi de 1996 a 2021, considerando a partir do ano de 1996 no qual foi sancionada a lei n. 9.394, de 20 de dezembro de 1996 que estabelece as diretrizes e bases da educação nacional e dispõe sobre a educação escolar e seus processos formativos predominantemente, por meio do ensino em instituições próprias.

Buscou-se as publicações indexadas no portal da biblioteca virtual em saúde - BVS (LILACS - Literatura LatinoAmericana e do Caribe em Ciências da Saúde) e na biblioteca digital SCIELO - Latino-Americana e do Caribe em Ciências da Saúde. Para a exclusão foram definidos os seguintes critérios: artigos duplicados, artigos de revisão e incompletos, teses e dissertações, livros, resenhas, estudos em escolas de outros países, em locais fora do âmbito escolar, bem como estudos que não traziam informações claras sobre a metodologia. A combinação de descritores, com base nos Descritores em Ciências da 
Saúde - DeCS, utilizados para a busca foram "Transtorno do déficit de atenção e hiperatividade", "crianças", "escolares", “docentes", "professores", utilizando na busca os operadores booleanos AND e OR. Para a seleção dos artigos, foi realizada uma leitura dos títulos e resumos.

$\mathrm{Na}$ terceira e quarta fases do estudo da revisão integrativa - Categorização e Avaliação dos estudos incluídos na revisão que se relacionam com o objetivo do estudo. Realizou-se a leitura dos periódicos e extração dos dados para serem compilados em um quadro, sumarizando a amostra que contemplaram os seguintes dados: Título do artigo, Autores, Ano, Local, População, Objetivos do estudo e Desfecho. Por fim, na quinta e sexta fase do estudo - Interpretação dos resultados e Síntese do conhecimento foram reunidos os dados do material coletado e organizado em duas categorias: a aprendizagem de crianças com TDAH e a percepção dos professores em relação ao transtorno.

\section{Resultados e Discussão}

Após o cruzamento dos descritores, obteve-se um resultado total de 646 artigos, sendo encontrados 238 na Literatura Latino-Americana e do Caribe em Ciências da Saúde (LILACS) e 408 na biblioteca Scientific Electronic Library Online (SCIELO). Após o critério de inclusão e exclusão e a realização da leitura do título e do resumo dos artigos, a amostra ficou em 44 artigos, sendo excluídos os que não atenderam aos objetivos do estudo, bem como os repetidos. Após a leitura na íntegra foram selecionados sete (07) artigos que abordavam o tema proposto e incluídos nesta revisão integrativa. A Figura 1 descreve o fluxograma da seleção dos artigos.

Figura 1: Fluxograma elaborado pelos autores com o número de artigos encontrados em cada base de dados por meio das buscas.

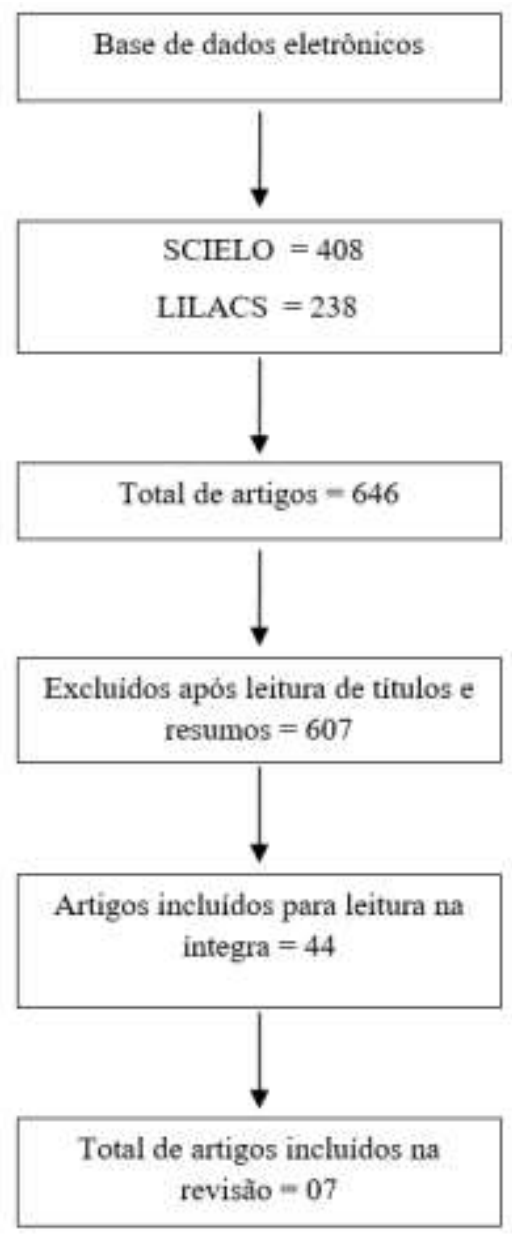

Fonte: Autores. 
As características sociodemográficas dos estudos selecionados estão apresentadas no Quadro 1, sendo possível verificar que somente na última década começaram a surgir estudos sobre a temática. Considerando o ano de publicação, a maioria dos estudos possuem menos de 5 anos, o que pode estar relacionado a data inicial da Lei Brasileira de Inclusão, também chamada de Estatuto da pessoa com deficiência, que afirmou a autonomia e a capacidade de cidadão a praticar atos da vida civil em condições de igualdade aos demais, bem como garantir direitos às pessoas pela nova lei (Brasil, 2015).

Considerando o local de realização do estudo, a maioria destes foi realizada no estado de São Paulo, o que evidencia a importância de outros estados brasileiros implementarem pesquisas acerca dessa temática na área da Educação em outras regiões do Brasil. A maioria dos estudos teve como amostra os escolares, sendo que três (estudos) realizaram um comparativo entre escolares com TDAH e escolares com bom desempenho acadêmico, indicando importantes resultados.

Quadro 1: Características sociodemográficas dos artigos, 2021.

\begin{tabular}{|c|c|c|c|c|}
\hline Autor & Título do artigo & Ano & Local & Amostra \\
\hline Jou et al. & $\begin{array}{l}\text { Transtorno de déficit de atenção e } \\
\text { hiperatividade: um olhar no ensino } \\
\text { fundamental }\end{array}$ & 2010 & Porto Alegre/RS & $\begin{array}{c}136 \text { professores de } 1^{\mathrm{a}} \text { à } 8^{\mathrm{a}} \\
\text { série }\end{array}$ \\
\hline Okuda et al. & $\begin{array}{l}\text { Função motora fina, sensorial e perceptiva } \\
\text { de escolares com transtorno do déficit de } \\
\text { atenção com hiperatividade }\end{array}$ & 2011 & Marília/SP & $\begin{array}{l}22 \text { escolares do ensino } \\
\text { fundamental }\end{array}$ \\
\hline Barini e Hage & $\begin{array}{l}\text { Vocabulário e compreensão verbal de } \\
\text { escolares com Transtorno do Déficit de } \\
\text { Atenção e Hiperatividade }\end{array}$ & 2015 & Bauru/SP & $\begin{array}{l}40 \text { crianças em idade } \\
\text { escolar }\end{array}$ \\
\hline $\begin{array}{c}\text { Silva, Santos e Oliveira } \\
\text { Filho }\end{array}$ & $\begin{array}{l}\text { Os significados do TDAH em discursos de } \\
\text { docentes dos anos iniciais }\end{array}$ & 2015 & Recife/PE & $\begin{array}{l}20 \text { professoras do ensino } \\
\text { fundamental }\end{array}$ \\
\hline $\begin{array}{l}\text { Uvo, Germano e } \\
\text { Capellini }\end{array}$ & $\begin{array}{l}\text { Desempenho de escolares com transtorno } \\
\text { do déficit de atenção com hiperatividade } \\
\text { em habilidades metalinguísticas, leitura e } \\
\text { compreensão leitora. }\end{array}$ & 2016 & Marília/SP & $\begin{array}{l}30 \text { escolares do Ensino } \\
\text { Fundamental I, de ambos } \\
\text { os gêneros, na faixa etária } \\
\text { de } 8 \text { anos a } 12 \text { anos e } 11 \\
\text { meses de idade }\end{array}$ \\
\hline $\begin{array}{l}\text { Inácio, Oliveira e } \\
\text { Mariano }\end{array}$ & $\begin{array}{l}\text { Estilos intelectuais e estratégias de } \\
\text { aprendizagem: percepção de professores } \\
\text { do ensino fundamental. }\end{array}$ & 2017 & Londrina/PR & $\begin{array}{l}23 \text { professores de escolas } \\
\text { públicas }\end{array}$ \\
\hline Paterlini et al. & $\begin{array}{l}\text { Triagem e diagnóstico de } \\
\text { dificuldades/transtornos de aprendizagem - } \\
\text { desfecho de avaliações interdisciplinares. }\end{array}$ & 2019 & Ribeirão Preto/SP & $\begin{array}{l}104 \text { crianças dos anos } \\
\text { iniciais do ensino } \\
\text { fundamental }\end{array}$ \\
\hline
\end{tabular}

Fonte: Autores (2021).

O Quadro 2 apresenta as características metodológicas dos estudos selecionados, evidenciando a utilização de Testes validados para a avaliação de habilidades linguísticas, perceptivo-motoras, cognitivas e de desempenho escolar. Esses estudos 
mostraram que os escolares com TDAH apresentam desempenho inferior aos escolares com bom desempenho acadêmico em relação às funções motoras fina, sensorial e perceptiva (Okuda et al., 2011), mostraram vocabulário mais restrito e mais dificuldades de compreensão verbal (Barini \& Hage, 2015), demonstram maior número de erros nas habilidades de identificação e manipulação de fonemas, de repetição de não palavras de maior extensão e de leitura de palavras e de não palavras (Uvo, Germano \& Capellini, 2016), sendo a disortografia a alteração de leitura e escrita mais prevalente, seguido por crianças classificadas como não alfabetizadas (Paterlini et al., 2019).

Ao analisar a percepção dos professores (Quadro 2), os estudos evidenciam que a maioria das crianças com o transtorno apresenta dificuldade para se concentrar, não termina as tarefas e são desatentas, qualificando as mesmas como agitadas, impulsivas, agressivas e com dificuldade de cumprir regras (Jou et al., 2010). Além disso, foi possível identificar a ausência de um significado unívoco para o termo TDAH, com uso de expressões que remetem a um repertório estritamente médico e à linguagem cotidiana, para dar sentido ao distúrbio (Silva, Santos \& Oliveira Filho, 2015), bem como que a maioria dos professores não conhecia termos como estratégias de aprendizagem e estilos intelectuais, por isso as respostas foram dadas de acordo com a sua prática de ensino (Inácio, Oliveira \& Mariano, 2017).

A Associação Americana de Psiquiatria (2014) aponta que esse transtorno possui como característica essencial um padrão persistente de desatenção e/ou hiperatividade-impulsividade que interfere no funcionamento/rotina da criança, sendo que o comportamento desatento está associado a vários processos cognitivos subjacentes, que podem repercutir em problemas cognitivos em testes de atenção, função executiva ou memória. 
Quadro 2: Características metodológicas dos artigos, 2021.

\begin{tabular}{|c|c|c|c|}
\hline Autor & Objetivo & Método & Desfecho \\
\hline Jou et al. & $\begin{array}{l}\text { Investigar o TDAH no } \\
\text { ambiente escolar, } \\
\text { considerando: quantidade de } \\
\text { casos e sua distribuição por } \\
\text { gênero; as medidas } \\
\text { terapêuticas mais utilizadas; } \\
\text { a percepção dos professores } \\
\text { acerca dos comportamentos } \\
\text { dos alunos; presença de } \\
\text { suporte profissional e } \\
\text { capacitação para os } \\
\text { professores nas escolas } \\
\text { estudadas. }\end{array}$ & Aplicação de questionários & $\begin{array}{l}\text { A média dos alunos com TDAH das escolas } \\
\text { estudadas foi de } 3 \% \text {. Em relação à percepção dos } \\
\text { professores, houve discrepância entre os possíveis } \\
\text { casos apontados por estes e os levantados junto à } \\
\text { direção. A maioria dos professores destacou como } \\
\text { características das crianças com o transtorno a } \\
\text { dificuldade para se concentrar, não terminar as } \\
\text { tarefas e ser desatentas, qualificando as crianças } \\
\text { como agitadas, impulsivas, agressivas e com } \\
\text { dificuldade de cumprir regras. Como } \\
\text { consequência dessas características todas, foram } \\
\text { citadas a baixa autoestima dos alunos e sua } \\
\text { exclusão pelos colegas. }\end{array}$ \\
\hline Okuda et al. & $\begin{array}{l}\text { Caracterizar e comparar as } \\
\text { funções motoras fina, } \\
\text { sensorial e perceptiva de } \\
\text { escolares com Transtorno do } \\
\text { Déficit de Atenção com } \\
\text { Hiperatividade (TDAH) e } \\
\text { escolares com bom } \\
\text { desempenho escolar sem } \\
\text { alterações de comportamento. }\end{array}$ & $\begin{array}{l}\text { Avaliação da Função Motora } \\
\text { Fina, Sensorial e Perceptiva } \\
\text { de dois grupos (GI = escolares } \\
\text { com TDAH; GII= escolares } \\
\text { com bom desempenho } \\
\text { acadêmico). }\end{array}$ & $\begin{array}{l}\text { Escolares com Transtorno de Déficit de Atenção } \\
\text { com Hiperatividade apresentam desempenho } \\
\text { inferior aos escolares com bom desempenho } \\
\text { acadêmico em relação às funções motoras fina, } \\
\text { sensorial e perceptiva. Tais dificuldades podem } \\
\text { causar impacto significativo sobre o desempenho } \\
\text { acadêmico, uma vez que comprometem o } \\
\text { desenvolvimento da linguagem escrita, } \\
\text { ocasionando disgrafia nesses escolares. }\end{array}$ \\
\hline Barini e Hage & $\begin{array}{l}\text { Comparar o vocabulário } \\
\text { receptivo e a compreensão } \\
\text { verbal de escolares com e sem } \\
\text { Transtorno do Déficit de } \\
\text { Atenção e Hiperatividade } \\
\text { (TDAH) e, ainda, confrontar o } \\
\text { desempenho deles com os } \\
\text { valores normativos dos testes } \\
\text { aplicados. }\end{array}$ & $\begin{array}{c}\text { Avaliação do vocabulário pelo } \\
\text { Teste de Vocabulário por } \\
\text { Figuras TVfusp e da } \\
\text { compreensão auditiva-verbal } \\
\text { pelo Token Test, versão } \\
\text { reduzida, em dois grupos (GE } \\
\text { = escolares com TDAH; GC= } \\
\text { escolares sem alterações de } \\
\text { aprendizagem, atenção e } \\
\text { linguagem }\end{array}$ & $\begin{array}{l}\text { Escolares com TDAH apresentaram vocabulário } \\
\text { mais restrito e mais dificuldades de compreensão } \\
\text { verbal quando comparados aos seus pares sem o } \\
\text { transtorno. O confronto do desempenho obtido } \\
\text { pelos grupos com os valores normativos do } \\
\text { TVfusp e do Token Test indicou que uma parcela } \\
\text { das crianças com TDAH apresenta desempenho } \\
\text { dentro da média nos testes aplicados. }\end{array}$ \\
\hline $\begin{array}{l}\text { Silva, Santos e } \\
\text { Oliveira Filho }\end{array}$ & $\begin{array}{l}\text { Investigar os significados do } \\
\text { TDAH (Transtorno do Déficit } \\
\text { de Atenção com } \\
\text { hiperatividade) em discursos } \\
\text { de docentes do ensino } \\
\text { fundamental, das redes } \\
\text { pública e privada, em } \\
\text { Pernambuco. }\end{array}$ & Entrevista semi-estruturada & $\begin{array}{l}\text { Ausência de um significado unívoco para o termo } \\
\text { TDAH, com uso de expressões que remetem a um } \\
\text { repertório estritamente médico e à linguagem } \\
\text { cotidiana, para dar sentido ao distúrbio. A maioria } \\
\text { deles descreveu o discente a partir de } \\
\text { características comportamentais, que ora enfocam } \\
\text { o excesso de atividade, ora centralizam a } \\
\text { desatenção. O estudo remete às dificuldades da }\end{array}$ \\
\hline
\end{tabular}




\begin{tabular}{|c|c|c|c|}
\hline & & & inserção e inclusão no espaço educacional. \\
\hline $\begin{array}{c}\text { Uvo, Germano e } \\
\text { Capellini }\end{array}$ & $\begin{array}{l}\text { Caracterizar e comparar o } \\
\text { desempenho de escolares com } \\
\text { Transtorno do Déficit de } \\
\text { Atenção com Hiperatividade } \\
\text { em habilidades } \\
\text { metalinguísticas, leitura e } \\
\text { compreensão leitora, com } \\
\text { escolares de bom desempenho } \\
\text { acadêmico }\end{array}$ & $\begin{array}{c}\text { Aplicação da Prova de } \\
\text { Habilidades Metalinguísticas } \\
\text { e de Leitura - PROHMELE } \\
\text { (Provas de identificação } \\
\text { silábica e fonêmica, Provas de } \\
\text { manipulação silábica e } \\
\text { fonêmica; Repetição de Não } \\
\text { Palavras; Provas de Leitura) } \\
\text { em dois grupos (GI=escolares } \\
\text { com TDAH; GII= escolares } \\
\text { com bom desempenho } \\
\text { acadêmico). }\end{array}$ & $\begin{array}{l}\text { Os escolares com TDAH apresentam maior } \\
\text { número de erros nas habilidades de identificação e } \\
\text { manipulação de fonemas, de repetição de não } \\
\text { palavras de maior extensão e de leitura de palavras } \\
\text { e de não palavras. Na prova de compreensão } \\
\text { leitora, ambos os grupos apresentaram } \\
\text { desempenho inferior, não havendo diferença } \\
\text { estatisticamente significante entre as populações } \\
\text { deste estudo, fazendo-nos refletir sobre as } \\
\text { condições de ensino da compreensão leitora em } \\
\text { contexto escolar. }\end{array}$ \\
\hline $\begin{array}{c}\text { Inácio, Oliveira e } \\
\text { Mariano }\end{array}$ & $\begin{array}{c}\text { Averiguar a percepção dos } \\
\text { professores acerca dos estilos } \\
\text { intelectuais e das estratégias } \\
\text { de aprendizagem em alunos } \\
\text { do Ensino Fundamental com } \\
\text { diagnóstico de Dislexia e } \\
\text { TDAH e sem dificuldade } \\
\text { escolar }\end{array}$ & Aplicação de questionário & $\begin{array}{l}\text { A maioria dos professores não conhecia termos } \\
\text { como estratégias de aprendizagem e estilos } \\
\text { intelectuais, por isso as respostas foram dadas de } \\
\text { acordo com a sua prática de ensino. }\end{array}$ \\
\hline Paterlini et al. & $\begin{array}{l}\text { Verificar a ocorrência do } \\
\text { baixo desempenho escolar em } \\
\text { crianças e investigar quais as } \\
\text { influências intrínsecas mais } \\
\text { prevalentes }\end{array}$ & $\begin{array}{c}\text { Avaliação de alterações } \\
\text { cognitivas pelo Mini-Exame } \\
\text { do Estado Mental adaptado } \\
\text { para criança (MEEM) e Teste } \\
\text { de Desempenho escolar em } \\
\text { crianças previamente } \\
\text { selecionadas por meio de uma } \\
\text { triagem }\end{array}$ & $\begin{array}{l}\text { Mais da metade das crianças em idade escolar } \\
\text { apresentaram déficits na aprendizagem da } \\
\text { linguagem escrita e/ou aritmética. As condições } \\
\text { mais prevalentes em crianças com baixo } \\
\text { desempenho escolar são as alterações de humor } \\
\text { (ansiedade e depressão) seguidas pela condição do } \\
\text { TDAH. Dentre as alterações de leitura, escrita e } \\
\text { aritmética, a disortografia é a mais prevalente, } \\
\text { seguido por crianças classificadas como não } \\
\text { alfabetizadas. }\end{array}$ \\
\hline
\end{tabular}

Fonte: Autores (2021).

A partir dos resultados dos estudos selecionados, emergiram duas categorias: a aprendizagem de crianças com TDAH e a percepção dos professores em relação ao transtorno. Tais categorias foram elencadas baseando-se na pertinência em identificar fatores de comprovação da aprendizagem em estudantes com o transtorno e expor a visão dos educadores perante desafios e estratégias para o êxito no processo de inclusão de estudantes com necessidades educacionais específicas.

\section{A aprendizagem das crianças com TDAH}

Após refinamento dos dados e classificação das categorias, a primeira categoria "aprendizagem de crianças com TDAH destaca três artigos os quais abordam as formas de aquisição de conteúdos por parte de estudantes do Ensino Fundamental I e II com o transtorno supracitado, bem como as estratégias e desafios no processo de ensino. 
O estudo de Uvo, Germano e Capellini (2016), visando caracterizar e comparar o desempenho de escolares com TDAH em habilidades metalinguísticas, leitura e compreensão leitora, com escolares de bom desempenho acadêmico, evidenciou que as crianças com esse transtorno apresentam, de uma forma geral, desempenho inferior em tarefas mais complexas, na decodificação leitora, nas tarefas metalinguísticas, especialmente em exercícios que demandam mais concentração, retenção de informações, análise e revisão de informações. Dessa forma, torna-se um desafio ao estudante os processos que demandam atenção exclusiva, fatores cognitivos relacionados à memória de trabalho, monitoramento e a capacidade de estabelecer inferências, ainda, fatores sociais e de ambiência tornam-se cruciais neste processo de obtenção do conhecimento (Andrade, 2006). Corroborando com a pesquisa anterior, o estudo de Paterlini et al. (2019), visando verificar os motivos do baixo rendimento escolar de crianças e as influências predominantes, evidenciou que $40 \%$ dos estudantes que possuíam baixo rendimento escolar apresentavam esse transtorno, determinando déficits em importantes domínios cognitivos necessários para o processo de alfabetização.

Em relação às características perceptivo-motoras, o estudo de Okuda et al. (2011), analisou dois grupos (GI = escolares com TDAH; GII = escolares com bom desempenho acadêmico), sendo que o GI apresentou desempenho inferior na função motora fina, sensorial e perceptiva. Esses déficits podem afetar a forma como ocorre o aperfeiçoamento da coordenação em atividades mais complexas como a utilização de ferramentas como o lápis e a tesoura, ou mesmo, na simples utilização de maneira mais independente das mãos e dedos (Trevisan, Coppede \& Capellini, 2008).

Nesse contexto, o estudo realizado por Zuanetti et al. (2016), realizado com prontuários de um ambulatório hospitalar, corrobora com os achados do presente estudo, pois indicam que mesmo com um nível intelectual dentro dos padrões médios as crianças com TDAH apresentaram bastante dificuldade de linguagem, bem como alterações emocionais e ambientais. Vale destacar que os autores apontaram que existe uma necessidade de atenção para essas crianças, pois mesmo sem comprometimento intelectual, apresentam graves dificuldades que comprometem o rendimento escolar, que pode apresentar níveis similares ou inferiores quando comparado às crianças com deficiência intelectual.

Nesse sentido, o DSM-V, da American Psychiatric Association (APA) que classifica os transtornos mentais, determina o TDAH como um transtorno do neurodesenvolvimento e de aprendizagem que se manifesta, geralmente, quando a criança ingressa na escola e, consequentemente, acarreta danos significativos no funcionamento pessoal, social, laboral e acadêmico (DSM-V, 2014). Reafirmando, existem evidências de que essas crianças possuem dificuldades na memória de trabalho e um desempenho inferior em relação às crianças sem os transtornos (Zuanetti et al, 2018).

Rohde et al. 2000, descrevem as características, bem como o perfil de comportamento de indivíduos que possuem a desordem a considerar a desatenção, hiperatividade e impulsividade, sobretudo, com manifestações como dificuldade de aterse a detalhes, erros em atividades escolares e profissionais simplesmente por descuido, e frequentemente suas respostas são precipitadas e não esperam a sua vez de falar interrompendo o assunto dos outros estando propensos a vários prejuízos no decorrer da vida acadêmica, social, familiar e profissional. Barini e Hage (2015), comparando a linguagem receptiva das crianças, um em nível de frases com ordem direta e outro em nível vocabular de dois grupos (GE = escolares com TDAH; $\mathrm{GC}=$ escolares sem alterações de aprendizagem, atenção e linguagem), encontrou desempenho inferior nas crianças do primeiro grupo, ressaltando os diferentes níveis de comprometimento, nos quais algumas crianças com o transtorno apresentam resultado dentro da média nos testes aplicados. Entretanto, de maneira geral, nas medidas de linguagem não foi comprovada diferença relevante entre GE e GC, mas crianças do GE demonstraram resultados piores no percentual de acertos para a identificação de palavras sinônimas.

\section{Percepção dos professores em relação ao TDAH}

Essa categoria revela questões que se mostram presentes na prática educacional vigente, além de expor fatos e 
discursos pautados no senso comum e até mesmo discriminatórios. Nessa perspectiva, o estudo de Inácio, Oliveira e Mariano (2017) analisando a percepção dos professores sobre estilos intelectuais e estratégias de aprendizagem, aponta que os educadores compreendem os benefícios dos estilos intelectuais para potencializar o aprendizado. Nesse estudo, quanto à estratégia, os docentes perceberam que seus alunos, de modo geral, buscavam se adaptar aos desafios impostos pelo transtorno, criando técnicas de assimilação em seus estudos, como sublinhar, anotar, pesquisar, reler e pedir ajuda.

O educador ao constatar algum tipo de necessidade educacional específica, pode auxiliar o aluno na identificação de estratégias (cognitivas e metacognitivas) que facilitem o processo de aprendizagem, além de proporcionar novas práticas e estabelecer objetivos a fim de garantir o desenvolvimento pessoal e intelectual deste aluno (Amaral, 2007). Apoiando-se no planejamento interventivo e em processos efetivos de construção do conhecimento, o artigo de Silva, Santos \& Oliveira Filho (2015) traz uma investigação de discursos, ideias e conceitos sobre TDAH a partir do olhar de professores do ensino fundamental das redes pública e privada em Pernambuco, refletindo sobre aspectos do modelo escolar vigente e manifesta as dificuldades da inserção e inclusão no espaço educacional. Ao analisar os discursos das docentes, os autores constatam um conjunto de contradições presentes em diferentes discursos e a relação com pressupostos ideológicos que regem suas práticas. As manifestações das professoras conduzem ainda a um foco de preocupação relacionado à disciplina, evidenciando discursos que relacionam o transtorno a situações de desvio social, ruptura da ordem escolar e percepções superficiais sobre dificuldades de aprendizagem. Ainda, algumas falas atribuem as dificuldades nas relações escolares exclusivamente à patologia da criança, evitando-se discutir e problematizar questões como políticas educacionais e práticas pedagógicas.

Na perspectiva de apontar posicionamentos de docentes sobre TDAH, o estudo de Jou et al. (2010), com professores de Porto Alegre, evidenciou que a maioria dos participantes reconhecem um distanciamento da teoria e da prática, especialmente no que tange à educação inclusiva. Vinculada a essa concepção, os dados mostraram que apenas $15 \%$ dos educadores apresentaram um conhecimento formal e mais aprofundado sobre o assunto na mesma linha. Assim, os resultados apontam para um número muito restrito de escolas que investem em cursos, palestras e algum tipo de suporte ao professor e ao estudante com esse transtorno.

Este fato reforça ainda mais a necessidade de investimentos em formação dos profissionais, o que é considerado como um dos dados mais relevantes do presente estudo, pois leva a refletir sobre quanto o posicionamento das escolas frente ao transtorno influencia na quantidade de casos diagnosticados e nas possibilidades de melhores adaptações pedagógicas. Considerando o exposto, é possível perceber que os artigos selecionados nas duas categorias discutem por meio de seus resultados a interferência do TDAH na aprendizagem das crianças e a importância de identificar os sintomas previamente, que são tão presentes na vida destes, e assim, obtendo um maior esclarecimento sobre as dificuldades enfrentadas por elas. A exemplo disso, Mattos (2016) afirma que devido às características do transtorno a criança apresenta dificuldades de aprendizagem e de relacionamento exigindo muita atenção para o desenvolvimento infantil.

Ainda cabe destacar que para os professores incentivarem os alunos no uso das estratégias de aprendizagem, é necessário que saibam valorizar o aprender e se apropriem de conhecimentos técnico-científicos relativos à aprendizagem, pois o despreparo para a intervenção junto aos alunos com estratégias de aprendizagem pode estar atrelado à déficits em sua formação (Santos \& Boruchovitch, 2011).

\section{Considerações Finais}

De modo geral, foi possível perceber que os estudos evidenciam que crianças com TDAH apresentam dificuldades de aprendizagem e baixo rendimento escolar. Tal distúrbio interfere, significativamente, no processo ensino-aprendizagem das crianças tornando uma barreira para um bom desempenho acadêmico.

Entende-se a importância, no cenário educacional, onde se concentram a maior parte das demandas por atendimentos 
especializados, o conhecimento prévio do transtorno por parte dos professores ao atuarem em sala de aula com alunos que os possuem, bem como compreendam as reais dificuldades enfrentadas por eles, assim poderão adotar estratégias e metodologias de ensino que favoreçam e estimulem as crianças a terem mais autonomia e autoconfiança possibilitando o desenvolvimento das potencialidades e uma melhor qualidade de vida.

Sugere-se a continuidade de estudos relacionados à aprendizagem de crianças com essa problemática, buscando investigar novos caminhos, metodologias e processos pedagógicos de caráter efetivo e inovador destinados à promoção de uma aprendizagem significativa que contribuirá para o desenvolvimento intelectual dos indivíduos. Além disso, recomenda-se prosseguir com pesquisas acerca das particularidades dos tratamentos existentes para o enfrentamento dessa patologia.

\section{Referências}

ABDA - Associação Brasileira do Déficit de Atenção (2020). Manual diagnóstico e estatístico dos transtornos mentais.

Álvarez-García D., Barreiro-Collazo S., Núnez, J. C. \& Dobarro, A. (2016). Validade e confiabilidade do Questionário de Ciber Agressão para Adolescentes (CYBA). The European Journal of Psychology Applied to Legal Context, 8 (1), 69-77.

Amaral, V. L. (2007). Estratégias e estilos de aprendizagem: a aprendizagem no adulto. In: Amaral, V. L. Psicologia da educação. Natal: EDUFRN. p. 1-20.

Andrade, M. W. C. L. \& Dias, M. G. B. B. (2006). Processos que levam à compreensão de textos. Psicologia Em Estudo, 11 (1), $147-154$.

APA - Associação Americana de Psiquiatria (2014). Transtornos do neurodesenvolvimento: transtorno do espectro autista. In: APA - Associação Americana de Psiquiatria. DSM 5 - Manual Diagnóstico e Estatístico de Transtornos Mentais. Ed. Artmed. p. 31-234.

Barbarini, T. A (2020). Corpos “mentes”, emoções: uma análise sobre TDAH e socialização infantil. Psicologia e Sociedade.

Barini, N. S. \& Hage, S. R. de V. (2015). Vocabulary and verbal comprehension of students with Attention Deficit Hyperactivity Disorder. Codas, 27(5), 446451.

Barkley, R. A (2020). Transtorno de Déficit de Atenção / Hiperatividade (TDAH) - Guia completo para pais, professores e profissionais da saúde. Artmed.

Biederman, J., Mesquinho, C. R., Woodworth, K. Y., Lomedico, U., Hyder, E. E. \& Faraone, S. V. (2012). Adulto resultado do déficit de atenção / hiperatividade transtorno: UMA controlada 16 anos acompanhamento estude. O Diário do Clínico Psiquiatria, 73(1), 941-950.

Billig, M. (2008). Argumentando e pensando: uma abordagem retórica à psicologia social. Vozes.

Brasil. (2015). Lei Brasileira de Inclusão da Pessoa com Deficiência (Estatuto da Pessoa com Deficiência). Brasília: presidência da república.

Cunha, V. L. O., Silva, C. da, Lourencetti, M. D., Padula, N. A. de M. R., \& Capellini, S. A. (2012). Desempenho de escolares com transtorno de déficit de atenção e hiperatividade em tarefas metalinguisticas e de leitura. Revista CEFAC, 15(1), 40-50. https://doi.org/10.1590/s1516-18462012005000003

Dalsgaard S., Nielsen H. S. \& Simonsen M (2014). Consequências do uso de medicamentos para TDAH nos resultados das crianças. Journal of Health Economics, 37(1), 137-151.

Jou, G. I., Amaral, B., Pavan, C. R., Schaefer, L. S., \& Zimmer, M. (2010). Attention deficit hyperactivity disorder: A glance at the elementary school. Psicologia: Reflexão e Crítica, 23(1), 29-36. https://doi.org/10.1590/S0102-79722010000100005

Fragoso, F. M. R. A., \& Casal, J. (2012). Representações sociais dos educadores de infância e a inclusão de alunos com necessidades educativas especiais. Revista Brasileira de Educação Especial, 18(3), 527-546. https://doi.org/10.1590/S1413-65382012000300011

German, J. R., Moore, D. A., Cooper, P., Russell, A. R., Richardson M., Morwenna R. \& Garside, R. (2016). A systematic review and synthesis of qualitative research: The influence of school context on symptoms of attention deficit hyperactivity disorder. Emot Behav Difficulties, 21(1), 83-100. https://doi.org/10.1080/13632752.2015.1120055

Gonçalves, J. P., \& Volk, M. (2016). Concepções das Professoras e Trabalho Educativo Voltado aos Alunos portadores de TDAH. Revista de Ensino, Educação e Ciências Humanas, 17(3), 220. https://doi.org/10.17921/2447-8733.2016v17n3p220-231

Inácio, F. F., de Oliveira, K. L., \& Mariano, M. L. S. (2017). Estilos intelectuais e estratégias de aprendizagem: Percepção de professores do ensino fundamental. Psicologia Escolar e Educacional, 21(3), 447-455. https://doi.org/10.1590/2175-3539/2017/021311171

Inete, M., Maia, R., \& Confortin, H. (2015). TDAH e aprendizagem: um desafio para a educação Hyperactivity and learning: an education challenge. Perspectiva, 39(148), 73-84. http://www.uricer.edu.br/site/pdfs/perspectiva/148_535.pdf

Larroca, L. M.; Domingos (2012), N. M. TDAH - Investigação dos critérios para diagnóstico do subtipo predominantemente desatento. Psicologia Escolar e Educacional, 16(1), 113-123.

Mattos, P. (2006). No mundo da lua: perguntas e respostas sobre o Transtorno do Déficit de Atenção com Hiperatividade em crianças, adolescentes e adultos. São Paulo: Lemos Editorial, 2006. 
Mendes, K. D. S., Silveira, R. C. de C. P., \& Galvão, C. M. (2008). Revisão integrativa: método de pesquisa para a incorporação de evidências na saúde e na enfermagem. Texto \& Contexto - Enfermagem, 17(4), 758-764. https://doi.org/10.1590/s0104-07072008000400018

Miranda A., Colomer C., Berenguer C., Roselló R., \& Roselló B (2016). Uso de substâncias em adultos jovens com TDAH: Comorbidade e sintomas de desatenção e hiperatividade / impulsividade. International Journal of Clinical and Health Psychology, 16(1), 157-165.

Miranda, U. M. A., Berenguer, C., Colomer, C., \& Roselló, R. (2014). Influência do a sintomas do Atenção Deficit Hyperactivity Transtorno (TDAH) e comórbido desordens em funcionando em idade adulta. Psicotema, 26(1).

Moreira, M. A. (1999). Teorias de Aprendizagem. EPU.

Moura, L. T., \& Silva, K. P. M. (2019). O Transtorno de Déficit de Atenção e Hiperatividade (TDAH) e as práticas pedagógicas em sala de aula. Revista Eletrônica Acervo Saúde, 22(1), e216.

Nogueira, M., Bosch, R., Valero, S., Gómez-Barros, N., Palo-março G., Richarte, V., Corrales, M., Nasillo, V., Vidal,R., Casas, M., \& Ramos-Quiroga, J. U. M. A. (2014). Early-age clinical and developmental features associated to Substance Use Disorders in Attention-Deficit/Hyperactivity Disorder in Adults. Comprehensive Psychiatry, (55)3, 639-649.

Pastura, G.; Mattos, P.; \& Araújo, A. P. Q. C. (2007) Prevalência do transtorno do deficit de atenção e hiperatividade e suas comorbidades em uma amostra de escolares. Arquivos de Neuropsiquiatria, 65(4) 1078-1083, 2007.

Paterlini, L. S. M., Zuanetti, P. A., Pontes-Fernandes, A. C., Fukuda, M. T. H., \& Hamad, A. P. A. (2019). Screening and diagnosis of learning disabilities/disorders - outcomes of interdisciplinary assessments. Revista CEFAC, 21(5). https://doi.org/10.1590/1982-0216/201921513319.

Polanczyk G. V., Willcutt E. G., Salum G. A., Kieling C., \& Rohde L. (2014). As estimativas de prevalência de TDAH ao longo de três décadas: Uma revisão sistemática atualizada e análise de meta-regressão. International Journal of Epidemiology, 43(1), 434-442.

Rohde, L. A., Miguel Filho, E. C., Benetti, L., Gallois, C., \& Kieling, C. (2004). Transtorno de déficit de atenção/hiperatividade na infância e na adolescência: considerações clínicas e terapêuticas. Arch. Clin. Psychiatry, 31(3), 124-131.

Rohde, L. A, Dorneles, B. V., \& Costa, A. C. (2006). Intervenções escolares no transtorno de déficit de atenção/hiperatividade. In: Rotta, N. T., Ohlweiler, L., \& Riesgo, R. S. (Eds.). Transtornos de aprendizagem: Abordagem neurobiológica e multidisciplinar. Artes Médicas. $365-374$.

Santos, O. J. X. \& Boruchovitch, E. (2011). Estratégias de aprendizagem e aprender a aprender: concepções e conhecimento de professores. Psicologia: Ciência e Profissão, 31(2), 284-295.

Santos, L. D. F; \& Vasconcelos, L. A. (2010). Transtorno do Déficit de Atenção e Hiperatividade em crianças: uma revisão interdisciplinar. Psicologia: teoria e pesquisa, 26 (4), 717-724.

Silva, K. V. P. da. (2015). Transtorno do déficit de atenção e hiperatividade (TDAH): Um olhar pedagógico. Revista Eventos Pedagógicos, 6(4), $223-231$.

Silva, M. L. V., Soares, N. S., Soares, R. S. C., Andrade, M. K. B. A., Ziane, S. R., \& Abreu, I. S. (2020). Abordagens em saúde mental em pessoas com transtorno de déficit de atenção com hiperatividade (TDAH): uma revisão integrativa. Research, Society and Development, 9(8), e628985933. http://dx.doi.org/10.33448/rsd-v9i8.5933

Silva, N. C., \& Carvalho, B. G. E. (2017). Compreendendo o processo de inclusão escolar no Brasil na perspectiva dos professores: Uma revisão integrativa. Revista Brasileira de Educação Especial, 23(2), 293-308. https://doi.org/10.1590/s1413-65382317000200010

Silva, S. P. da, Santos, C. P., \& Oliveira Filho, P. de. (2015). Os significados do TDAH em discursos de docentes dos anos iniciais. Pro-Posições, 26(2), 205221. https://doi.org/10.1590/0103-7307201507708

Okuda, P. M. M., Pinheiro, F. H., Germano, G. D., Padula, N. A. M. R., Lourencetti, M. D., Cristina, L., \& Capellini, S. A. (2011). Função motora fina, sensorial e perceptiva de escolares. Jornal da Sociedade Brasileira de Fonoaudiologia, 1(3), 351-357.

Trevisan J. G., Coppede A. C., \& Capellini S. A (2008). Avaliação da função motora fina, sensorial e perceptiva em escolares com dificuldades de aprendizagem. Temas Desenvol, 16(94), 183-187.

Zuanetti, P. A., Santos, K. A. S., Nascimento, F. M., \& Fukuda, M. T. H. (2016). Desempenho escolar de crianças com distúrbio de aprendizagem: comparação entre crianças com e sem deficiência intelectual TT - School performance of children with learning difficulties: comparison between children with and without intellectual disabiliti. Distúrb. Comun, 28(2), 202-210. http://revistas.pucsp.br/index.php/dic/article/view/22791/20132

Zuanetti, P. A., Lugli, M. B., Fernandes, Â. C. P., Soares, M. da S. T., Silva, K. da, \& Fukuda, M. T. H. (2018). Memory performance, oral comprehension and learning process between children with attention deficit hyperactivity disorder and children with anxiety disorder. Revista CEFAC, 20(6), 692-702. https://doi.org/10.1590/1982-0216201820614218

Zucchetti, D. T. (2011). A inclusão escolar vista sob a ótica de professores da escola básica. Educação em Revista, 27(2), 197-218. https://doi.org/10.1590/s0102-46982011000200010 